\title{
Screening in Hot SU(2) Gauge Theory and Propagators in 3d Adjoint Higgs model
}

\author{
A. Cucchieri, F. Karsch and P. Petreczky \\ Fakultät für Physik, Universität Bielefeld, P.O. Box 100131, D-33501 Bielefeld, Germany
}

\begin{abstract}
We investigate the large distance behavior of the electric and magnetic propagators of hot SU(2) gauge theory in different gauges using lattice simulations of the full $4 \mathrm{~d}$ theory and the effective, dimensionally reduced $3 \mathrm{~d}$ theory. A comparison of the $3 \mathrm{~d}$ and $4 \mathrm{~d}$ data for the propagators suggests that dimensional reduction works surprisingly well down to temperatures $T \sim 2 T_{c}$. A detailed study of the volume dependence of magnetic propagators is performed. The electric propagators show exponential decay at large distances in all gauges considered and a possible gauge dependence of the electric screening mass turns out to be statistically insignificant.
\end{abstract}

The poles of the finite temperature gluon propagator can be related to screening of chromoelectric and chromomagnetic fields 11. The static chromoelectric (Debye) screening mass was calculated in leading order of perturbation theory long ago and was found to be gauge independent to this order [1]. The existence of a static chromomagnetic screening mass generated nonperturbatively was postulated by Linde to render the perturbative expansion of different thermodynamic quantities finite [2]. Beyond the leading order also the Debye screening mass is non-perturbative [3], i.e depends explicitly on the magnetic mass. Static electric and magnetic propagators and the corresponding screening masses were studied in $\mathrm{SU}(2)$ gauge theory in Landau gauge [4. In Ref. [5] it was shown that static propagators could be studied in the effective dimensionally reduced version of finite temperature $\mathrm{SU}(2)$ gauge theory, the $3 \mathrm{~d}$ adjoint Higgs model. Here we will discuss the determination of the screening masses from propagators calculated in different gauges and their gauge dependence. Results calculated within full 4d SU(2) gauge theory as well as the effective $3 \mathrm{~d}$ theory will be presented.

In four dimensions (4d) all our calculations are performed with the standard Wilson action for $\mathrm{SU}(2)$ lattice gauge theory. We will use the notation $\beta_{4}=4 / g_{4}^{2}$ for the lattice gauge coupling. In three dimensions the standard dimensional reduction process leads us to consider the $3 \mathrm{~d}$ adjoint
Higgs model

$$
\begin{gathered}
S=-\beta_{3} \sum_{P} \frac{1}{2} \operatorname{Tr} U_{P} \\
-\beta_{3} \sum_{\mathbf{x}, \hat{\mu}} \frac{1}{2} \operatorname{Tr} A_{0}(\mathbf{x}) U_{\mu}(\mathbf{x}) A_{0}(\mathbf{x}+\hat{\mu}) U_{\mu}^{\dagger}(\mathbf{x})+ \\
\frac{1}{4} \beta_{3} \sum_{\mathbf{x}}\left[(6+h) \operatorname{Tr} A_{0}^{2}(\mathbf{x})+x\left(\operatorname{Tr} A_{0}^{2}(\mathbf{x})\right)^{2}\right],
\end{gathered}
$$

where $\beta_{3}$ now is related to the dimensionfull $3 d$ gauge coupling and the lattice spacing a, i.e. $\beta_{3}=4 / g_{3}^{2} a$, and the adjoint Higgs field is parameterized by hermitian matrices $A_{0}=\sum_{a} \sigma^{a} A_{0}^{a}\left(\sigma^{a}\right.$ are the usual Pauli matrices). Furthermore, $x$ parameterizes the quartic self coupling of the Higgs field and $h$ denotes the bare Higgs mass squared. We also note that the indices $\mu, \nu$ run from 0 to 3 in four dimensions and from 1 to 3 in three dimensions.

As we want to analyze properties of the gluon propagator, which is a gauge dependent quantity, we have to fix a gauge on each configuration on which we want to calculate this observable. In the past most studies of the gluon propagator have been performed in Landau gauge. Here we will consider a class of $\lambda$-gauges introduced in Ref. [6]. In the continuum these gauges correspond to the gauge condition

$\lambda \partial_{0} A_{0}+\partial_{i} A_{i}=0$.

Here the index $i$ runs from 1 to 3 . The case $\lambda=1$ corresponds to the usual Landau gauge. In addition to the $\lambda$-gauges we also consider the Maximally Abelian gauge (MAG) 77. In this case 
one has to fix a residual gauge degree of freedom which we do by imposing an additional U(1)Landau gauge condition [8]. In the $4 \mathrm{~d} \mathrm{SU}(2)$ gauge theory we also consider the static time averaged Landau gauge (STALG) introduced in Ref.[9]. In continuum it is defined by

$\partial_{0} A_{0}\left(x_{0}, \mathbf{x}\right)=0, \quad \sum_{x_{0}} \sum_{i=1}^{3} \partial_{i} A_{i}=0$.

While the notion of Landau and Maximally Abelian gauges carries over easily to the $3 \mathrm{~d}$ case we have to specify our notion of $\lambda$-gauges in $3 d$. We have considered two versions of $\lambda$-gauge,

$\partial_{1} A_{1}+\partial_{2} A_{2}+\lambda_{3} \partial_{3} A_{3}=0$,

$\lambda_{1} \partial_{1} A_{1}+\partial_{2} A_{2}+\partial_{3} A_{3}=0$,

which we will refer to as $\lambda_{1}$-gauge and $\lambda_{3}$-gauge correspondingly. Furthermore, we consider in $3 \mathrm{~d}$ the Coulomb gauge, which fixes the gauge in a plane transverse to the $z$-direction, $\partial_{1} A_{1}+\partial_{2} A_{2}=$ 0 . The residual gauge freedom is fixed by demanding that $\sum_{x, y} U_{3}(\mathbf{x})=u_{30}$ should be constant.

All our $4 \mathrm{~d}$ simulations have been performed at temperature $T=2 T_{c}$. The values for the $4 \mathrm{~d}$ coupling $\beta_{4}$ corresponding to this temperature were taken from Ref. [4], $\beta_{4}=2.52$ for $N_{t}=4$ and $\beta_{4}=2.74$ for $N_{t}=8$. The $3 \mathrm{~d}$ adjoint Higgs model simulations were done for three sets of parameters, $\beta_{3}=11, x=0.099, h=-0.395$ corresponding to $T=2 T_{c}$, and $\beta_{3}=16, x=0.03$, $h=-0.2085$ as well as $\beta_{3}=24, x=0.03$, $h=-0.1510$ which correspond to $T \sim 9200 T_{c}$. For the $3 \mathrm{~d}$ gauge coupling $g_{3}^{3}$ we always use the 1loop relation $g_{3}^{2}=g^{2}(T) T$ with $g(T)$ being the $4 \mathrm{~d}$ 1-loop running coupling constant in $\overline{M S}$-scheme with $\bar{\mu}=18.86 T$ 12. The detailed procedure for fixing the parameters of the $3 \mathrm{~d}$ effective theory is described in Ref. [12].

We note that for static fields the gauge condition for $4 \mathrm{~d} \lambda$-gauges, $\lambda \partial_{0} A_{0}+\partial_{i} A_{i}=0$ is equivalent to $3 \mathrm{~d}$ Landau gauge. The same is true for STALG. One would expect that if dimensional reduction works, propagators calculated in different $\lambda$-gauges and STALG agree with each other, and of course, with the propagators calculated in the
3 d effective theory in Landau gauge. A comparison of the corresponding data in $4 \mathrm{~d} \mathrm{SU}(2)$ gauge theory and the $3 \mathrm{~d}$ effective theory shows that this is indeed the case. Furthermore, we have compared the electric and magnetic propagators calculated in $4 \mathrm{~d} \mathrm{SU}(2)$ gauge theory and $3 \mathrm{~d}$ effective theory in MAG. Good agreement between 3d and $4 \mathrm{~d}$ data was found also here.

Previous lattice calculations of the magnetic propagator in hot $\mathrm{SU}(2)$ gauge theory in $4 \mathrm{~d}$ 顿 and in the $3 \mathrm{~d}$ adjoint Higgs model $[5]$ gave evidence for its exponential decay in coordinate space and thus suggested the existence of a magnetic mass. In was also found that the magnetic propagators calculated in the $3 \mathrm{~d}$ adjoint Higgs model are quite insensitive to the scalar couplings and are quite close to the corresponding propagators of $3 \mathrm{~d}$ pure gauge theory. In Ref. [11] the Landau gauge gluon propagator of $3 \mathrm{~d}$ gauge theory was studied in momentum space and was found to be infrared suppressed for large volumes. Such a behavior clearly rules out the existence of a simple pole mass. To clarify the picture of magnetic screening a detailed study of finite size effects is necessary. In what follows we will mainly concentrate on a discussion of the magnetic propagators in the limit of the $3 \mathrm{~d}$ pure gauge theory. Where appropriate, comparison with the results from $4 \mathrm{~d} \mathrm{SU}(2)$ gauge theory will be made. In Figure 17a we show the magnetic propagators in coordinate space on different volumes at $T=2 T_{c}$. Calculations were done in $4 \mathrm{~d} \mathrm{SU}(2)$ gauge theory at $\beta_{4}=2.52,2.74$ and in $3 \mathrm{~d}$ pure gauge theory at $\beta_{3}=8$. On small volumes the propagator indeed shows exponential decay but it continues to drop faster as the volume increases. For volumes $V T^{3} \gtrsim 330$ we have clear evidence that the propagators become negative at $z T \gtrsim 2$. A similar strong volume dependence was observed in other $\lambda$-gauges and also in Coulomb gauge.

Because of the strong volume dependence of the magnetic propagators in $\lambda$-gauges simulations on large lattices are necessary to get control over finite size effects. We have analyzed the magnetic propagators in Landau gauge in the $3 \mathrm{~d}$ pure gauge theory for $\beta_{3}=5$ and on lattices of size $L^{3}$ with $L=32,40,48,56,64,72$ and 96 . In what follows all dimensionfull quantities will be scaled by ap- 
propriate powers of the $3 \mathrm{~d}$ gauge coupling $g_{3}$. Using the relation of $g_{3}$ to the renormalized 4 d gauge coupling (see above) it is straightforward to express dimensionfull quantities in units of $T$. The magnetic propagators at $\beta_{3}=5$ and for different volumes are shown in Figure $1 \mathrm{~b}$ and Figure 1c. As one can see from the figure the volume dependence of the coordinate space propagator is small on these large lattices. The propagator becomes negative for $z g_{3}^{2} \gtrsim 4$. The strong volume dependence translates into infrared sensivity of the momentum space propagators. For $p / g_{3}^{2}<0.3$ the momentum space propagator is sensitive to the volume, while for large momenta $\left(p / g_{3}^{2}>0.3\right)$ it is essentially independent of the lattice size. Moreover, we note that for small momenta the finite volume effects lead to a decrease of $\tilde{D}(p)$ with increasing volume while the volume dependence is already negligible for $p / g_{3}^{2} \simeq 0.3$. Thus the magnetic propagators in Landau gauge are infrared suppressed. The propagators in other $\lambda$-gauges show similar behavior. Let us note that the infrared suppression of the Landau gauge propagator was observed also $T=04 \mathrm{~d} \mathrm{SU}(\mathrm{N})$ gauge theory [13].

In contrast to the complicated structure found in Landau gauge the propagator calculated in MAG does show a simple exponential decay at large distances and does not show any significant volume dependence. We find for the magnetic screening mass in MAG $m_{M}=0.50(5) g_{3}^{2}$.

Contrary to the magnetic propagators the electric propagators show little volume dependence and decay exponentially in all gauges considered. We have investigated in detail the gauge dependence of the electric propagators in the $3 \mathrm{~d}$ effective theory at temperature $T \sim 9200 T_{c}$. The results are summarized in Figure 2. As one can see from this figure any possible gauge dependence of the local electric masses in the region where they reach a plateau is statistically insignificant.

Acknowledgments: The work has been supported by the TMR network ERBFMRX-CT970122 and by the DFG under grant Ka 1198/4-1. The numerical calculations have partly been performed at the HLRS in Stuttgart and the $(P C)^{2}$ in Paderborn.

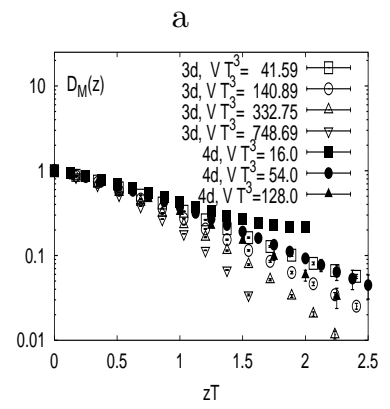

$\mathrm{b}$

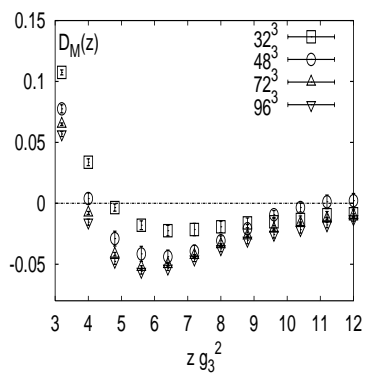

c

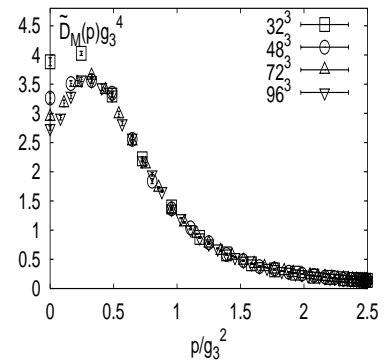

Figure 1. The magnetic gluon propagators in Landau gauge. Shown are the magnetic propagators in coordinate space from $4 \mathrm{~d}$ simulations and from $3 \mathrm{~d}$ pure gauge theory at $\beta_{3}=8$ (a), the magnetic propagators in coordinate space at large distances calculated in 3d pure gauge theory at $\beta_{3}=5(\mathrm{~b})$ and the momentum space magnetic propagators from $3 \mathrm{~d}$ pure gauge theory at $\beta_{3}=5$ (c). The coordinate space propagators were normalized to 1 at $z=0$. 


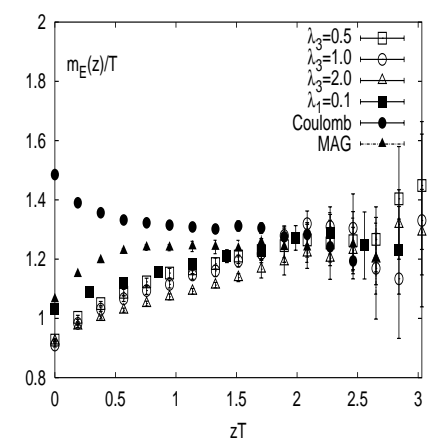

Figure 2. Local electric masses calculated in $\lambda$ gauges, Coulomb gauge and MAG at $T \sim 9200 T_{c}$. Calculation were done in the $3 \mathrm{~d}$ effective theory at $\beta_{3}=24, x=0.03$ and $h=-0.1510$ except in the case of $\lambda_{1}=0.1$ where they were done at $\beta_{3}=16, x=0.03, h=-0.2085$.

\section{REFERENCES}

1. D.J. Gross et al., Rev. Mod. Phys. 5343 (1981)

2. A. Linde, Phys. Lett. B96 289 (1980)

3. A. Rebhan, Nucl. Phys. B430 319 (1994); P. Arnold and L.G. Yaffe, Phys Rev. D52 7208 (1995)

4. U.M. Heller et al., Phys. Lett. B355 511 (1995); Phys. Rev. D57 1438 (1998)

5. F. Karsch et al., Phys. Lett. B442 291 (1998)

6. C. Bernard et al., Nucl. Phys. B (Proc. Suppl.) 17593 (1990); Nucl. Phys. B (Proc. Suppl.) 20410 (1991)

7. A. S. Kronfeld et al., Phys. Lett. B198 516 (1987)

8. K. Amemiya and H. Suganuma, Phys.Rev. D60 114509 (1999)

9. C. Curci et al., Z. Phys. C26 549 (1985); T. Reisz, J. Math. Phys. 32515 (1991)

10. A. Cucchieri and F. Karsch Nucl. Phys. B (Proc. Suppl.) 83357 (2000)

11. A. Cucchieri, Phys. Rev. D60 034508 (1999)

12. A. Cucchieri, F. Karsch and P. Petreczky, in preparation

13. L. von Smekal et al., Phys. Rev. Lett. 79 (1997) 3591, D.B. Leinweber et al., Phys. Rev. D58 (1998) 0315011 